\title{
Functional Outcomes of Gastrocnemius Fascial Turn-down Flap with FHL Augmentation in Chronic Achilles Tear: A Short-term Prospective Study
}

\author{
Pradeep K Meena ${ }^{1}$, Nagaraj Manju Moger ${ }^{2}$, J Pragadeeshwaran ${ }^{3}$, Sukhmin Singh ${ }^{4}$
}

\begin{abstract}
Introduction: Tendo-Achilles (TA) is one of the main tendons utilized for every movement of the lower limb. Rupture of TA leads to severe disruption of overall mobility and leads to difficulty in doing daily routine activities. The purpose of this prospective study was to observe the functional outcome of gastrocnemius fascial turn down flap with flexor hallucis longus (FHL) augmentation for chronic TA tear.

Materials and methods: Tendo-Achilles rupture was diagnosed in all patients either by clinical or radiological means (most of them were type II or type III according to KUWADA classification). Preoperative functional scores (modified RUPP, FADI, FAAM, VAS) were documented. All 13 patients were treated surgically by gastrocnemius fascial turn down flap with FHL augmentation. All surgeries were performed by the same surgeon. The standard postoperative protocol was followed in all patients. Patients were followed up regularly at 3 months, 6 months, 1 year, and 2 years postoperative for functional outcome score.

Results: The results were statistically analyzed by paired $T$-test for evaluating improvement in pre- and post-intervention periods. Functional outcome scores showed a significant improvement with FAAM score was improved from $30.84 \pm 2.9$ to $76.23 \pm 3.98$ ( $p<0.001$ ), FADI $40.92 \pm 3.15$ to $85.2 \pm 4.04(p<0.001)$, modified RUPP score at 2 weeks postoperatively was $3.07 \pm 1.18$ which at 2 years of follow-up improved to mean score $22.77 \pm 3.45(p<0.0001)$. VAS preoperatively value of $7.77 \pm 1.01$ improvised to $1.85 \pm 0.99$ at 2 years' follow-up ( $p<$ 0.01 ) showing a significant decrease in overall pain.

Conclusion: Functional outcomes show turn down flap with FHL augmentation as a good procedure for chronic TA rupture. Proper rehabilitation and wound care is the mainstay for good outcomes. Wound dehiscence is, however, a challenge that needs special care for providing good outcomes.

Level of evidence: III

Keywords: Foot and ankle ability measure, Foot and ankle disability index, RUPP score, Tendo-Achilles, Visual analog scale.

Journal of Foot and Ankle Surgery (Asia Pacific) (2022): 10.5005/jp-journals-10040-1201
\end{abstract}

\section{INTRODUCTION}

Achilles tendon (TA) is formed by joined tendons of plantaris, gastrocnemius, and soleus muscle. ${ }^{1}$ It transmits weight from the triceps surae (combined two-headed gastrocnemius and the soleus) to the heel (by calcaneal attachment). Being the thickest and strongest tendon in the human body, it can withstand the bodyweight of up to eight times as during running. ${ }^{2-4}$ Degeneration or trauma can lead to the rupture of TA on its course. Tendo-Achilles tear usually occurs $4-6 \mathrm{~cm}$ proximal to its calcaneal insertion due to its anatomical reason (hypovascular). ${ }^{2}$

Depending on the duration, TA tear can be classified as acute or chronic. Acute Achilles tendon rupture (AATR) is most commonly seen among athletes as they are commonly involved in running, jumping, and sprinting movements to make TA at risk of rupture. ${ }^{5,6}$ ATTR can be treated either by non-operative or operative depending upon the grade of rupture, wound status, the general condition of the patient. Non-operative treatment with splinting (casting) is associated with good results. Surgical repair technique for ATTR is preferred due to the decreased rate of re-rupture and faster recovery. ${ }^{7-9}$ Wound complication is a challenge to the surgeon in operative treatment. A rupture that has been untreated for 6 weeks or more is called a chronic TA rupture. ${ }^{10,11}$ Chronic TA rupture can be treated either conservatively by casting and orthosis or surgically by an end-to-end repair with or without flexor hallucis longus (FHL) augmentation, turn down flap, and with

\footnotetext{
1,3 Department of Orthopaedics, All India Institute of Medical Sciences, Rishikesh, Uttarakhand, India

${ }^{2}$ Department of Orthopaedics, Vardhman Mahavir Medical College and Hospital, Safdarjung, New Delhi, India

${ }^{4}$ Department of Orthopaedics, Gautam Budha Chikitsa Mahavidyalya, Dehradun, Uttarakhand, India
}

Corresponding Author: Sukhmin Singh, Department of Orthopaedics, Gautam Budha Chikitsa Mahavidyalya, Dehradun, Uttarakhand, India, Phone: +91 9805177633, e-mail: sukhmin92@gmail.com

How to cite this article: Meena PK, Moger NM, Pragadeeshwaran J, et al. Functional Outcomes of Gastrocnemius Fascial Turn-down Flap with FHL Augmentation in Chronic Achilles Tear: A Short-term Prospective Study. J Foot Ankle Surg (Asia Pacific) 2022;9(1):16-20.

Source of support: Nil

Conflict of interest: None

VY advancement. ${ }^{12,13}$ For the management of TA rupture in 1990, KUWADA proposed a classification based on the severity of tear and retraction of cut ends, which groups TA injury into four types. ${ }^{14}$

Numerous studies have been reported until now in the literature about different types of TA injury and its management along with their outcomes. ${ }^{5,6}$ Literature supports surgical management as a better modality of treatment for TA rupture given re-rupture and improved functional outcome. ${ }^{15}$ 
The purpose of this study is to analyze functional outcomes after surgical management of TA rupture with turn down flap and FHL augmentation following chronic insertional tendo-Achilles (TA) rupture.

\section{Materials and Methods}

A prospective study was conducted at a Tertiary care center in North India from February 2017 to October 2019. A total of 13 patients who met inclusion criteria were included in this study. Inclusion criteria were age between 18 years and 60 years, injury hospital duration of 6 weeks or more, no history of local steroid infiltration, the gap between ruptured ends of $>3 \mathrm{~cm}$. The patients were diagnosed by both clinical (Palpation and Simmonds-Thompson test) and radiological examination (USG or MRI). Most of our study patients were either type II or type III of KUWADA classification. Most of the patients had sedentary lifestyles except one who was a laborer. Eight patients reported to our hospital within 5 months of injury, the earliest was within 1 month of injury and the longest duration was 1 year. Preoperative VAS and functional scores were documented. All 13 patients were treated surgically with gastrocnemius fascial turn down flap and FHL augmentation. All surgeries were done by the same surgeon. All patients were discharged on postoperative day 2 with anterior below knee slab applied in 5-10 degrees of dorsiflexion. Skin sutures were removed on postoperative day 14 and splintage was done in neutral ankle dorsiflexion. Suture removal was delayed in one patient because of wound-related complications. Patients were allowed to weight bear gradually from partial to full weight after 4 weeks and the cast was removed after 6 weeks postoperative. Patients were followed up regularly up to 2 years postoperative for functional outcome scores. Foot and Ankle Ability Measure (FAAM), ${ }^{16}$ Foot and Ankle Disability Index (FADI), ${ }^{17}$ Visual Analog Scale (VAS), ${ }^{18}$ and modified RUPP score ${ }^{19}$ were measured and analyzed preoperatively and at last, follow-up after 2 years postoperative.

\section{Surgical Procedure}

All patients were positioned prone after spinal anesthesia. A wellpadded pneumatic tourniquet was applied at the thigh and inflated to $150 \mathrm{~mm} \mathrm{Hg}$ above systolic blood pressure. Parts were painted and draped following universal precautions. A $10-15 \mathrm{~cm}$ incision was made posteromedial to TA. A skin incision was deepened to reach the ruptured end of the tendon (Fig. 1). Para-Tenon was incised in line with skin to expose cut edges and degenerative tendon edges were excised (Fig. 2). The gap (G) was analyzed in neutral ankle dorsiflexion. $2 \times(G+2) \mathrm{cm}$ central gastrocnemius fascial flap was marked (Fig. 3). This flap was rotated down and was sutured to the remaining stump on the calcaneum with sutures using modified giftbox technique (Fig. 4). A long FHL graft was harvested proximal to Knot of Henry by directly giving $2 \mathrm{~cm}$ incision placed over plantar aspect at the level of the navicular bone and it was passed from medial to the lateral aspect of calcaneum by creating a tunnel and the loop was sutured over TA repair for augmentation (Fig. 5). After a thorough wound wash sutures were applied in layers with special attention to the closure of para-Tenon (Fig. 6).

\section{Statistical Analysis}

Categorical variables were presented as numbers. Continuous variables were analyzed with mean and standard deviation. Paired $T$-test and Pearson's correlation were used. A $p$ value of 0.05 or less was considered significant.
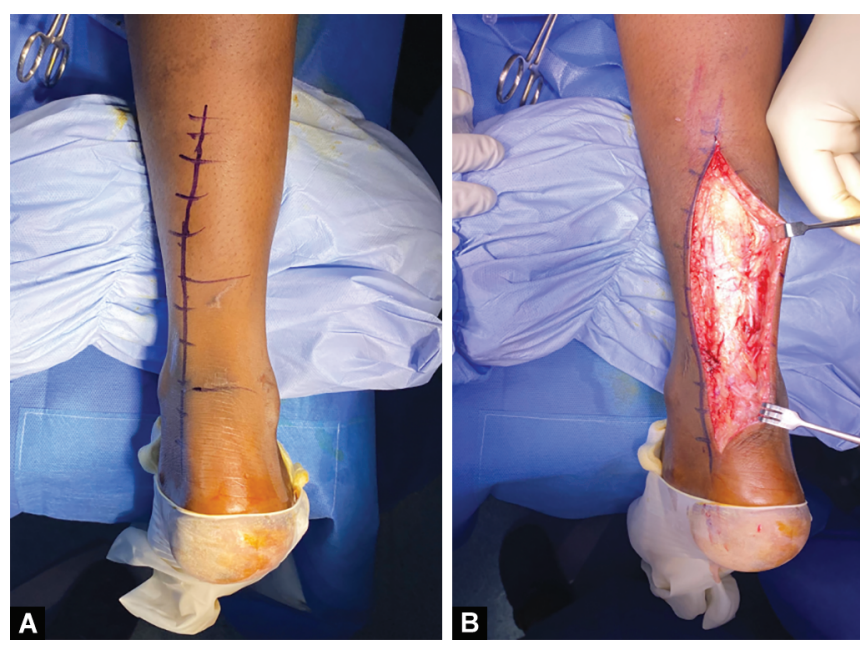

Figs $1 \mathrm{~A}$ and $\mathrm{B}$ : (A) Positioning and marking of incision; (B) Skin incision

\section{Results}

A total of 13 patients were included in the study, 5 females and 8 males, with an average age of 42.6 years \pm 6.6 (mean \pm SD), minimum being 27 years and maximum being 51 years. The average injury hospital duration is 5.9 months. All patients returned to their routine daily activities at an average of $10.7 \pm 1.22$ weeks (Table 1).

The mean preoperative FAAM score was $30.84 \pm 2.9$ which improved to a mean score of $76.23 \pm 3.98$ at 2 years' follow-up ( $p$ $<0.001)$. The mean FADI score preoperative was $40.92 \pm 3.15$ which at 2 years' follow-up was $85.2 \pm 4.04$ ( $p<0.001)$. Modified RUPP score at 2 weeks postoperatively was $3.07 \pm 1.18$ which at 2 years' follow-up improved to mean score of $22.77 \pm 3.45$ ( $p<0.0001)$.

VAS improved from a preoperative value of $7.77 \pm 1.01$ to $1.85 \pm 0.99$ at 2 years' follow-up $(p<0.01)$ (Table 2$)$.

\section{Discussion}

Chronic tendinopathy is a degenerative condition of the watershed area of TA which is $2-6 \mathrm{~cm}$ area from the insertion at calcaneum. ${ }^{20}$ Spontaneous rupture is very common in such conditions or with a slight traumatic injury. Following rupture, patients usually complain of gait dysfunction, persistent debilitating pain which often hampers their daily routine activities. ${ }^{15,21}$ There are several ways in treating a chronic tear which are described in the literature. Debriding the residual tissue and clearing ends with tendon transfer had been well described. Debridement leads to a larger gap formation which warrants a tendon transfer in most of the cases. ${ }^{22}$ In our study, we performed a gastrocnemius fascial turn down flap with FHL augmentation with good outcomes.

Flexor hallucis longus is an excellent option for transfer in TA rupture as it has the axis of pull parallel to TA along with good contractile force and both being the in-phase muscles. ${ }^{23}$ Our findings demonstrate significant improvement in functional outcomes, decrease in pain, and return to daily routine activities. Koh et al. demonstrated a similar outcome in turn down flap with FHL augmentation. ${ }^{21}$ They described the only difference in the groups with and without turn down flap as operative time with no statistical difference in functional outcomes. They, however, emphasized the importance of increased operative time period in turn down flap groups associated with complications. In our study, comparatively only one patient had wound-related complications 

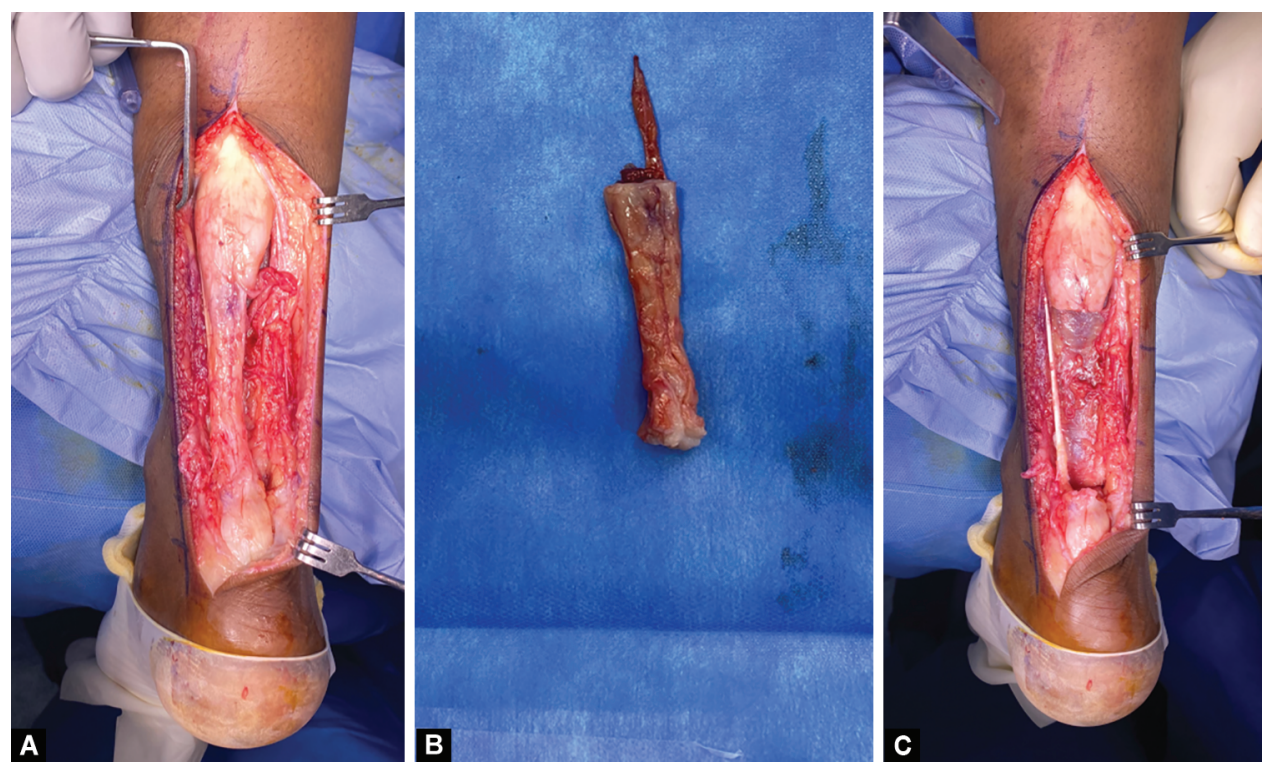

Figs 2A to C: (A) Exposed tendon; (B) Excised tendon; (C) After tendon removal
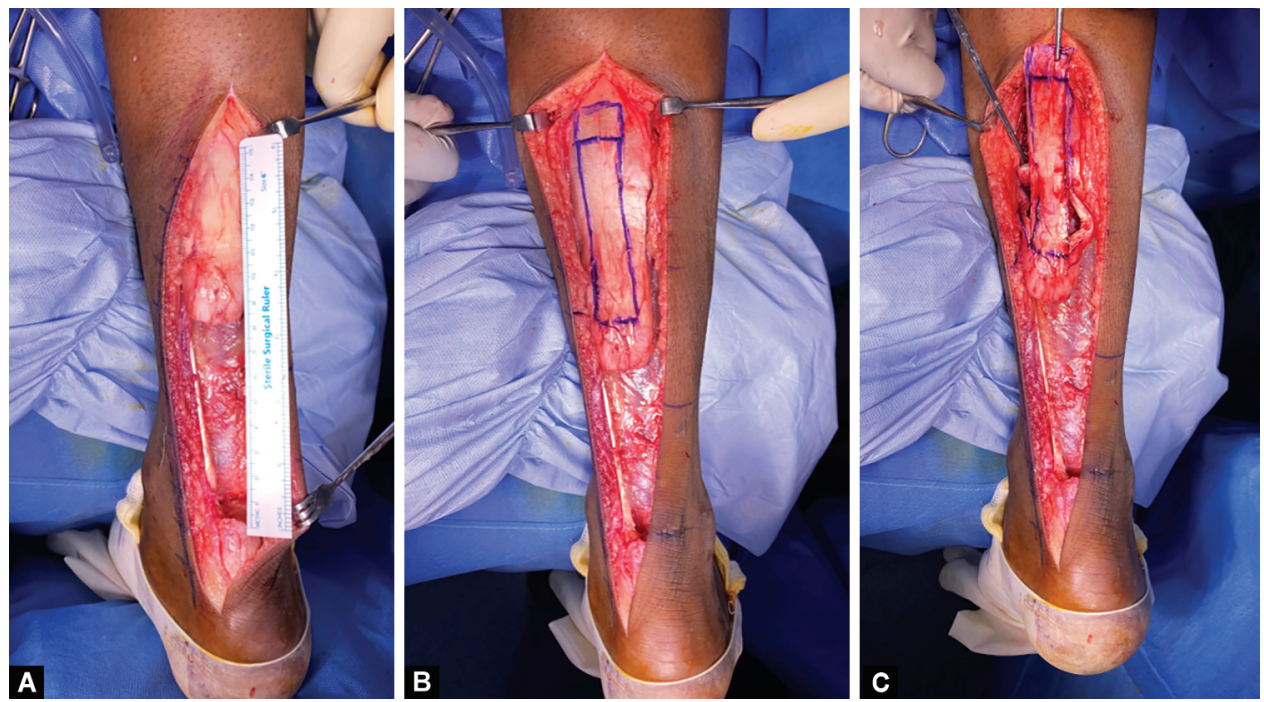

Figs 3 A to C: (A) Planning of the flap after measuring gap (G); (B) Flap markings done $(2 \times(G+2))$; $(C)$ Flap raised according to planned margins

which were addressed successfully with antibiotics and regular dressings.

El-Tantawy and Azzam described good results in their series of 13 patients in which they modified the procedure. They used FHL as the main material in-between the gap and turned down the flap forward. The mean improvement in AOFAS was $>70 \%(p<0.001)$ with 11 patients having excellent results and 2 good results. ${ }^{24}$

The majority of the studies had used AOFAS being the major functional outcome score for the follow-up but as its validity is now in question, ${ }^{25}$ we utilized other scoring systems which are well established. FAAM, FADI, modified RUPP, and VAS improved significantly at 2 years' follow-up $(p<0.05)$. VAS score showed that there was a significant improvement in the pain postoperatively. The average time period for return to routine activities was 10.7 weeks with all patients finally returning to their pre-injury activity levels.
Our study has a good follow-up period (2 years) with multiple outcome scores utilized for analyzing the subjective as well as objective satisfaction of the operative procedure. Various limitations with our study include the small sample size. Secondly, no comparative analysis of the functional outcome was done with other methods of TA repair. Lastly, the gait analysis which will assess the overall improvement in the mobility of the patients was not done.

Randomized control studies are necessary with a large cohort to compare the various operative procedures. Additionally, gait analysis should be done to look out for the overall improvement which is a big lacuna in most of the studies regarding the TA rupture.

\section{Conclusion}

Gastrocnemius fascial turn down flap with FHL augmentation provides a good outcome in patients with chronic Achilles 

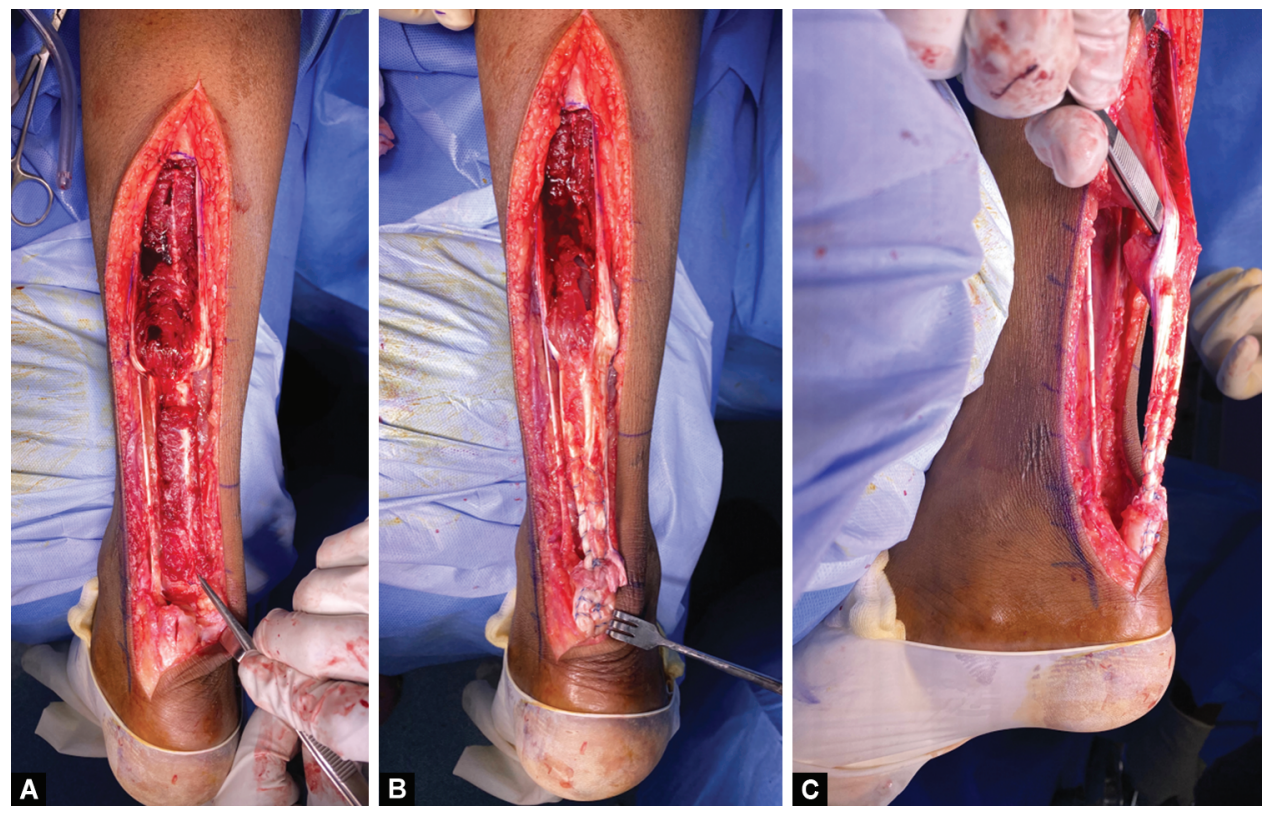

Figs 4A to C: (A) Flap turned down; (B) Sutured tendon; (C) Final continuous tendon
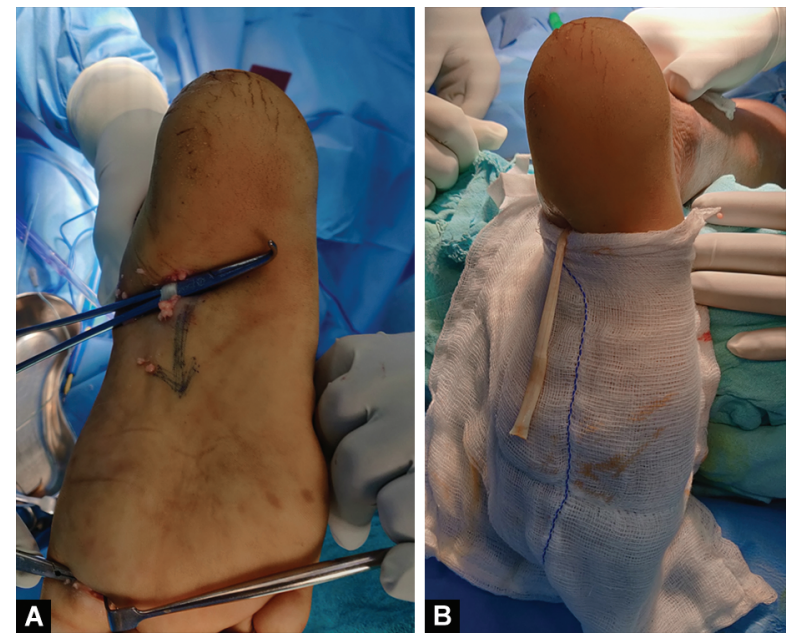

Figs 5A and B: (A) FHL identified at knot of Henry; (B) Tendon released

Table 1: Demographic details

\begin{tabular}{ll}
\hline Total patients & 13 \\
\hline Gender & Male -8 \\
& Female -5 \\
Age in years (mean \pm SD) & $42.6 \pm 6.6$ \\
Injury hospital duration (mean in & $5.9121 \pm 6.91749$ \\
months \pm SD) & \\
KUWADA classification & Type I-I \\
& Type II-V \\
& Type III-VII \\
Return to routine activities & $10.7 \pm 1.22$ weeks \\
Complications & Wound dehiscence - 1 \\
\hline
\end{tabular}

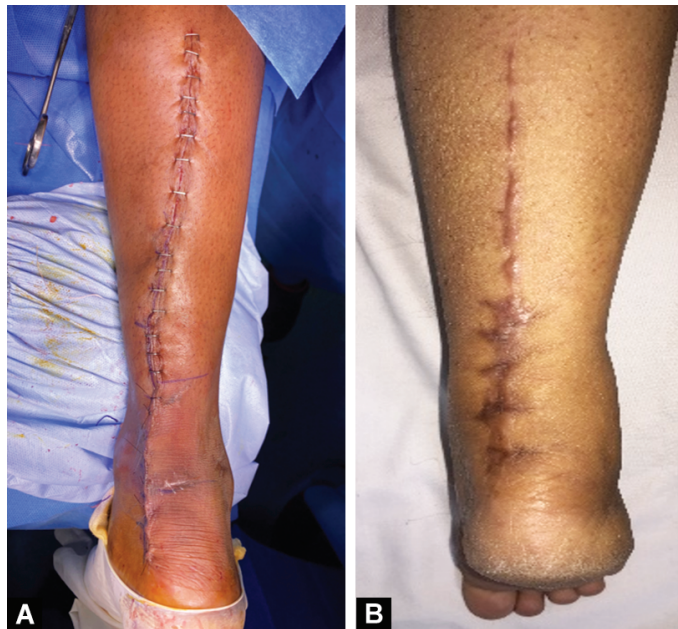

Figs $6 \mathrm{~A}$ and B: (A) Final closure; (B) Scar after 6 months postoperative

tendinopathy. Surgical management poses challenges at the various fronts for orthopedic surgeons in this area with wound complications being the most common. A proper rehabilitation with wound care proves to be beneficial for earlier return to activities with good patient satisfaction rates.

\section{Ethical Approval}

This article does not contain any studies with human participants or animals performed by any of the authors.

\section{InFormed CONSENT}

Informed consent was obtained from all individual participants included in the study. 
Turn-down Flap with FHL Augmentation for Chronic Achilles Tear with Various Functional Outcome Scores

Table 2: Functional outcome scores

\begin{tabular}{|c|c|c|c|c|c|}
\hline Functional score & Mean & Std. dev. & Std error mean & $\begin{array}{l}\text { Pearson's correlation } \\
\text { value }\end{array}$ & $\begin{array}{l}p \text { value (two-tailed } \\
\text { correlation) }\end{array}$ \\
\hline FAAM preoperative & 30.84 & 2.91 & 0.807 & 0.578 & $<0.001$ \\
\hline Postoperative & 76.23 & 3.98 & 1.104 & & \\
\hline FADI preoperative & 40.92 & 3.14 & 0.875 & 0.422 & $<0.001$ \\
\hline Postoperative & 85.2 & 4.04 & 1.119 & & \\
\hline Modified RUPP & & & & 0.268 & $<0.001$ \\
\hline $\begin{array}{l}2 \text { weeks postopera- } \\
\text { tive }\end{array}$ & 3.07 & 1.18 & 0.329 & & \\
\hline 2 years postoperative & 22.77 & 3.45 & 0.961 & & \\
\hline VAS preoperative & 7.77 & 1.01 & 0.281 & 0.462 & $<0.001$ \\
\hline Postoperative & 1.85 & 0.99 & 0.273 & & \\
\hline
\end{tabular}

\section{References}

1. Gabel S, Mandi A. Foot fellow's review neglected rupture of the achilles tendon,. Foot Ankle Int 1994(9):512-517. DOI: 10.1177/107110079401500912.

2. Kumar DKS, Babu R, Shaik T, et al. Merits and demerits of different surgical techniques in treating old, neglected ruptures of tendo achilles. Int J Orthop Sci 2018;4(1c):190-194. DOI: 10.22271/ortho.2018. v4.i1c.30.

3. Paavola $M$, Kannus $P$, Paakkala $T$, et al. Long-term prognosis of patients with achilles tendinopathy: an observational 8-year follow-up study. Am J Sports Med 2000;28(5):634-642. DOI: 10.1177/03635465000280050301.

4. Jung HG. Foot and ankle disorders: an illustrated reference. Foot Ankle Disord An Illus Ref 2016;28(1):1-630. DOI: 10.1007/978-3-64254493-4.

5. Willits K, Amendola A, Bryant D, et al. Operative versus nonoperative treatment of acute achilles tendon ruptures: a multicenter randomized trial using accelerated functional rehabilitation. J Bone Jt Surg - Ser A 2010;92(17):2767-2775. DOI: 10.2106/JBJS.I. 01401.

6. Carmont MR, Heaver C, Pradhan A, et al. Surgical repair of the ruptured achilles tendon: the cost-effectiveness of open versus percutaneous repair. Knee Surgery, Sport Traumatol Arthrosc 2013;21(6):1361-1368. DOI: 10.1007/s00167-013-2423-1.

7. Chana JS, Chen HC, Jain V. A new incision for surgery on tendo Achillis using a distally-based fasciocutaneous flap. J Bone Jt Surg - Ser B 2002;84(8):1142-1144. DOI: 10.1302/0301-620X.84B8.13405.

8. Čretnik A, Kosanović M, Smrkolj V. Percutaneous versus open repair of the ruptured Achilles tendon: a comparative study. Am J Sports Med 2005;33(9):1369-1379. DOI: 10.1177/0363546504271501.

9. Dalton GP, Wapner KL, Hecht PJ. Complications of Achilles and posterior tibial tendon surgeries. Clin Orthop Relat Res 2001;391(391):133-139. DOI: 10.1097/00003086-20011000000014.

10. Ebinesan AD, Sarai BS, Walley GD, et al. Conservative, open or percutaneous repair for acute rupture of the Achilles tendon. Disabil Rehabil 2008;30(20-22):1721-1725. DOI: 10.1080/09638280701786815.

11. Jiang N, Wang B, Chen A, et al. Operative versus nonoperative treatment for acute Achilles tendon rupture: a meta-analysis based on current evidence. Int Orthop 2012;36(4):765-773. DOI: 10.1007/ s00264-011-1431-3.

12. Aibinder WR, Patel A, Arnouk J, et al. The rate of sural nerve violation using the achillon device: a cadaveric study. Foot Ankle Int 2013;34(6):870-875. DOI: 10.1177/1071100712473097.
13. Bradley JP, Tibone JE. Percutaneous and open surgical repairs of Achilles tendon ruptures: a comparative study. Am J Sports Med 1990;18(2):188-195. DOI: 10.1177/036354659001800213.

14. Distefano V. Ruptures of the achilles tendon. AthI Train 1975;10(4):195198.

15. Spennacchio $P$, Vascellari $A$, Cucchi $D$, et al. Outcome evaluation after Achilles tendon ruptures. A review of the literature. Joints 2016;4(1):52-61. DOI: 10.11138/jts/2016.4.1.052.

16. Martin RL, Irrgang JJ, Burdett RG, et al. Evidence of validity for the foot and ankle ability measure (FAAM). Foot Ankle Int 2005;26(11):968-983. DOI: 10.1177/107110070502601113.

17. Hale SA, Hertel J. Reliability and sensitivity of the foot and ankle disability index in subjects with chronic ankle instability. J Athl Train 2005;40(1):35.

18. Delgado DA, Lambert BS, Boutris $\mathrm{N}$, et al. Validation of digital visual analog scale pain scoring with a traditional paper-based visual analog scale in adults. J Am Acad Orthop Surg Glob Res Rev 2018;2(3):e088. DOI: 10.5435/JAAOSGlobal-D-17-00088.

19. Kerkhoffs GMMJ, Struijs PAA, Raaymakers ELFB, et al. Functional treatment after surgical repair of acute Achilles tendon rupture: wrap vs walking cast. Arch Orthop Trauma Surg 2002;122(2):102-105. DOI: 10.1007/s004020100312.

20. Baindoor P, Gumaste A, Jeevannavar S, et al. Full thickness open tear of the tendoachilles - functional outcomes following a stable, single stage repair. J Clin Orthop Trauma 2021;14(January 2012):132-138. DOI: 10.1016/j.jcot.2020.06.033.

21. Koh D, Lim J, Chen JY, et al. Flexor hallucis longus transfer versus turndown flaps augmented with flexor hallucis longus transfer in the repair of chronic Achilles tendon rupture. Foot and Ankle Surgery 2019;25(2):221-225. DOI: 10.1016/j.fas.2017.10.019.

22. Ozer H, Ergisi $Y$, Harput $G$, et al. Short-term results of flexor hallucis longus transfer in delayed and neglected Achilles tendon repair. J Foot Ankle Surg 2018;57(5):1042-1047. DOI: 10.1053/j.jfas.2018.03.005.

23. Lever CJ, Bosman HA, Robinson AHN. The functional and dynamometer-tested results of transtendinous flexor hallucis longus transfer for neglected ruptures of the Achilles tendon at six years' follow-up. Bone Jt J 2018;100-B(5):584-589. DOI: 10.1302/0301620X.100B5.BJJ-2017-1053.R1.

24. El-Tantawy A, Azzam W. Flexor hallucis longus tendon transfer in the reconstruction of extensive insertional Achilles tendinopathy in elderly: an improved technique. Eur J Orthop Surg Traumatol 2015;25(3):583-590. DOI: 10.1007/s00590-014-1569-y.

25. Sharma S. The AOFAS clinical rating systems-time to bid adieu? J Foot Ankle Surg (Asia Pacific) 2019;6(1):1-1. DOI: 10.5005/ jp-journals-10040-1103. 